
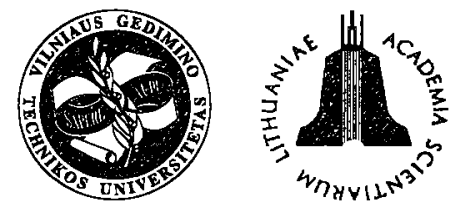

ISSN 1648-4142 TRANSPORT

http//www.vtu.lt/english/editions

TRANSPORT - 2003, Vol XVIII, No 3, 103-107

\title{
THE APPLICATION OF A FUZZY ALGORITHM FOR THE DETERMINATION OF THE SHORTEST CHAIN TO CORRECT DISORGANIZED TRAIN TRAFFIC
}

\author{
Robertas Stadalius, Vaclovas Bagdonas \\ Dept of Control Technology, Kaunas University of Technology, Studentu g. 48-320, \\ LT-3031 Kaunas, Lithuania.E-mail:Vaclovas.Bagdonas@ktu.lt
}

Received 200301 03; accepted 20030430

\begin{abstract}
The problem of the correction of the disorganized train iraffic schedule is analyzed (aiming to minimize general costs related to the expenditure of energy and time wasted by people and equipment). Disorganized a train traffic is named an event when you should insert one more train route in the already created train traffic schedule. This problem can be solved applying the algorithms for the search of the shortest chain The fuzzy algorithm for the determination of the shortest chain, the key operators and their specific features are also analyzed.
\end{abstract}

Keywords: traffic control; railway; mathematical model; shortest chain; uncertainty; algorithm.

\section{Introduction}

A train traffic schedule is the whole complex of routes (planned for a certain period), when exact arrival time at every station $t_{j i}^{a}$ and departures from it $t_{j i}^{d}$ are provided for every train $(j-$ train index, $i=1, n-$ station index). The arrival and departure time of trains $j, \beta$ and $(j+1)$ is planned and specified in Fig 1. In this fragment of the traffic schedule trains $j$ and $(j+1)$ are deemed as "planned" ones (their routes in advance had to be planned economically in optimum). Meanwhile the train $\beta$ could "appear" unplanned: it appeared in this place of the traffic schedule through some faults or organizational difficulties or it became urgently necessary to insert one more route into the already prepared traffic schedule, etc.

The problem arises to make such a schedule of the further train $\beta$ traffic that would guarantee minimum additional economic expenditures.

\section{Algorithm of the Disorganized Train Traffic cor- rection}

If there is a semi automatic blocking (here only this case shall be analyzed) equipped in the section, for the arrival of the unplanned train $(\operatorname{train} \beta)$ at the station $S_{(i+1)}$, a time span of which is remained with the width of

$$
\Delta=t_{(j+1) i}^{d}-t_{j(i+2)}^{a} .
$$

If the train $\beta$ is late at the station $S_{i+1}$, it can retard the train $(j+1)$ (will force it to stop at the station $S_{i}$ ). The train $\beta$ can also be forced to stop at the station $S_{i+1}$ in a similar way (if the train $\mathrm{j}$ is late for the station $S_{i+2}$ ).

Every additional stooping of the train is related to certain losses that appear through additional expenditures of fuel (energy) when the train is making its run. Losses are experienced at the station $S_{i}$ upon forced stopping of the train $(j+1){ }^{h i} W_{(j+1) i}$, and while stopping the train $\beta$ at the station $S_{i+1}$-as ${ }^{s t} W_{\beta(i+1)}$. Additional train $\beta$ may also experience losses while standing at the station $S_{i}$ and waiting for more favourable traffic situation (in order it will not be stopped and will not impede the train following it). These waiting losses (until $r$ number of

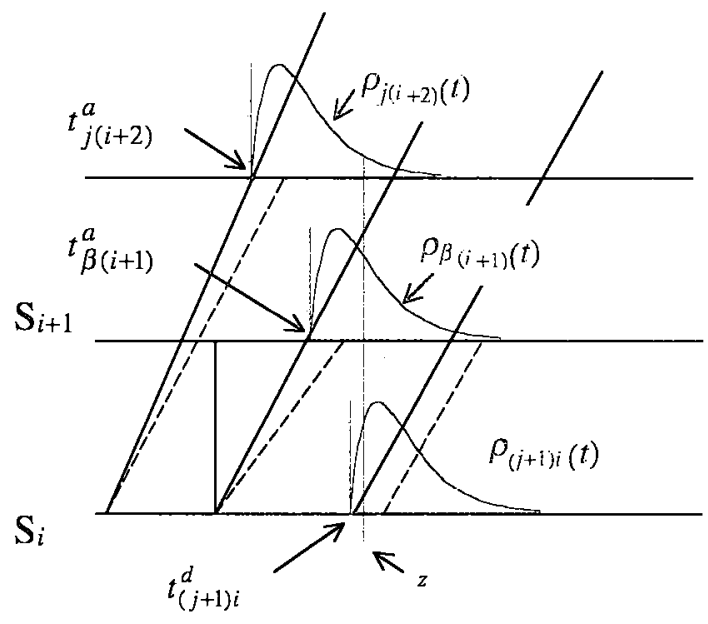

Fig 1. Illustration explaining the problem of correction of the train traffic schedule 
trains will pass: from the train $(j+1)$ to $(j+r)$ inclusively) are marked as $W_{W j(j+r) i}$. The Methods of the calculation of all mentioned losses are known, they can be found in special literature [1].

The problem arises to make such a schedule of the further train $\beta$ traffic that would guarantee minimum additional economic expenditures

$$
V=\sum_{j, r, i} W_{W j(j+r) i}+\sum_{j, i}^{h i} W_{j i}+\sum_{i}^{s t} W_{i}
$$

Practice shows that it is rather difficult to realize planned traffic schedules precisely through the manifestation of road and rolling-stock faults or disorders, meteorological conditions, organizational difficulties, etc. Thus solving similar problems by regular methods the solution is found not for actual, but for planned (desirable) initial data. Such solution often appears to be inadequate and may cause new traffic disorganization.

Then a new need to estimate the said unpredictable factors appears. Thus the regular problem of the traffic schedule correction turns into the statistical one. It is formed considering the delay of the following train and the stopping of the unplanned train $(\beta)$ as accidental events.

The researches show that delays of trains (processsing statistical data of several months) are rather adequately described by gamma distribution laws that are related to the second-line law of Erlang:

$$
\begin{aligned}
& \rho(t \mid \lambda, \eta=2)=\lambda^{2} t e^{-\lambda t}, \\
& t \geq 0, \quad \lambda>0 .
\end{aligned}
$$

Parameter $\lambda$ is different for every station, direction and train group (freight trains, passenger trains). Under certain circumstances many factors influencing a traffic schedule may be provided more precisely, but it is impossible to accumulate statistical data describing all possible cases. Through this, a traffic schedule correction problem appears to be a mixed one: it is purposeful to carry out a part of calculations applying regular methods, another part - statistical ones, and the other - expert (fuzzy) methods.

The problem of planning the optimum route of the additional train $\left(\beta^{\text {th }}\right)$ (according to the summed up economic expenditures V) leads to the problem of the search of the shortest chain in the specific graph of the rectangle network type. Such graph is shown in Fig 2.

Every vertex shown in Fig 2 is marked with a double index. The first element of this index specifies the train followed by the additional train $(\beta)$; the second element of the vertex specifies the station.

Vertical arcs correspond the movement of the additional train from one station to the other one and horizontal arcs - waiting (letting the following trains go).

Arc indices specify statistical costs related to corresponding transition.

$$
\begin{aligned}
& V_{M j i}={ }^{h i} P_{(j+1) i}{ }^{h i} W_{(j+1) i}+{ }^{s t} P_{\beta(i+1)}{ }^{s t} W_{\beta(i+1)}+ \\
& { }^{h i \& s t} P_{j i}\left({ }^{h i} W_{(j+1) i}+{ }^{s t} W_{\beta(i+1)}\right) .
\end{aligned}
$$

For every stage $\left(S_{i}-S_{i+1}\right)$ it is possible to find statistically optimal planned arrival time ${ }^{o p t} t_{\beta(i+1)}^{a}$ of the additional train $(\beta)$ at the station $(i+1)$ following the train $j$. This time can be found from the condition:

$$
\underset{d t_{\beta(i+1)}^{a}}{d} V_{M j i}\left(t_{\beta(i+1)}^{a}\right)=0 .
$$

Then (see Fig 1)

$$
\begin{aligned}
& { }^{h i} P_{(j+1) i}=\int_{t_{\beta(i+1)}^{a}}^{\infty} \rho_{\beta(i+1)}(z)\left(\int_{t_{j(i+2)}^{a}}^{z} \rho_{j(i+2)}(t) d t \times\right. \\
& \left.\quad \int_{t_{(j+1) i}^{i}}^{z} \rho_{(j+1) i}(y) d y\right) d z \\
& { }^{s t} P_{\beta(i+1)}=\int_{t_{\beta(i+1)}^{a}}^{\infty} \rho_{\beta(i+1)}(z)\left(\int_{z}^{\infty} \rho_{j(i+2)}(t) d t \times\right. \\
& \left.\int_{z}^{\infty} \rho_{(j+1) i}(y) d y\right) d z \text {. } \\
& \rho_{\beta(i+1)}(t) \text { in the formulas (6) and (7) means the }
\end{aligned}
$$

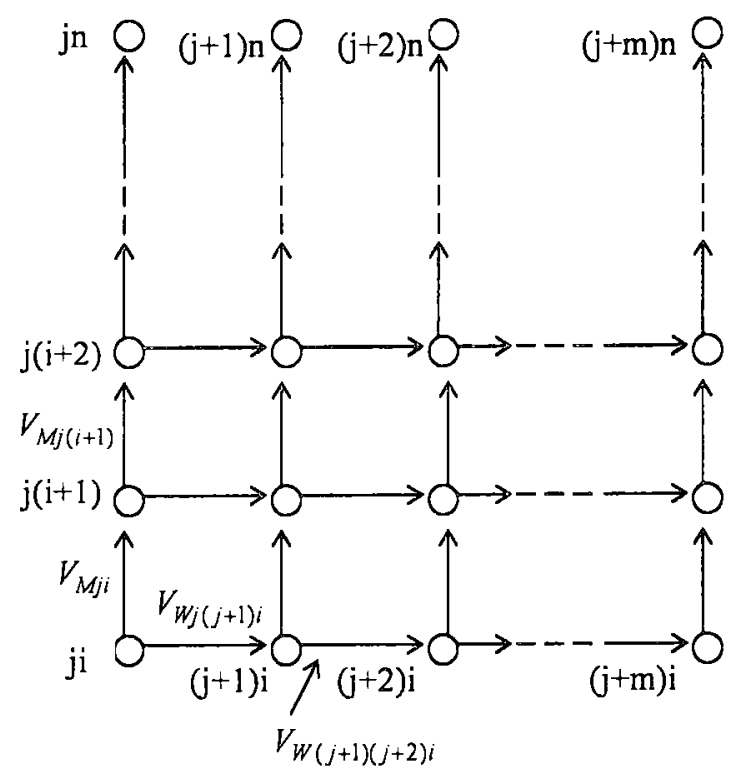

Fig 2. Graph of optimization of the route of the additional train $(\beta)$ 
probability distribution function of the delay of the train $\beta$ to the station $(i+1)$ (deviation from the planned time of the arrival at this station). All other functions of $\rho(t)$ (with different indices) have also the meaning of the probability distribution function. It is assumed that delays of trains are described by means of the formula (3), the parameter $\lambda$ of which is specified by the expert (traffic regulator, etc.) Thus $\rho(\mathrm{t})$ functions are fuzzy ones. ${ }^{h i} P_{(j+1) i}$, ${ }^{s t} P_{\beta(i+1)}$, as well as $V_{M j i}$ also turns into blank numbers:

$$
\begin{aligned}
& { }^{h i} P_{(j+1) i}=\exp \left(-\lambda_{\beta}\left(t_{(j+1) i}^{d}-t_{\beta(i+1)}^{a}\right)\right) \times \\
& \left(1-\left(\begin{array}{c}
\lambda_{\beta} \\
\lambda_{\beta}+\lambda_{j+1}
\end{array}\right)^{2}-\frac{2\left(\lambda_{\beta}\right)^{2} \lambda_{j+1}}{\left(\lambda_{\beta}+\lambda_{j+1}\right)^{3}}\right)+ \\
& \left(t_{(j+1) i}^{d}-t_{\beta(i+1)}^{a}\right) \exp \left(-\lambda_{\beta}\left(t_{(j+1) i}^{i}-t_{\beta(i+1)}^{a}\right)\right) \times \\
& \left(\begin{array}{cc}
\left(\lambda_{\beta}\right)^{2} & \left(\lambda_{\beta}\right)^{2} \lambda_{j+1} \\
\lambda_{\beta}-\lambda_{\beta}+\lambda_{j+1}- & \left(\lambda_{\beta}+\lambda_{j+1}\right)^{2}
\end{array}\right) . \\
& { }^{s t} P_{\beta(i+1)}=\exp \left(-\lambda_{j}\left(l_{\beta(i+1)}^{a}-t_{j(i+2)}^{a}\right)\right) \times \\
& \left(1-\left(\begin{array}{c}
\lambda_{j} \\
\lambda_{j}+\lambda_{\beta}
\end{array}\right)^{2}-\frac{2\left(\lambda_{j}\right)^{2} \lambda_{\beta}}{\left(\lambda_{j}+\lambda_{\beta}\right)^{3}}\right)+ \\
& \left(t_{\beta(i+1)}^{a}-t_{j(i+2)}^{a}\right) \exp \left(-\lambda_{j}\left(t_{\beta(i+1)}^{a}-t_{j(i+2)}^{a}\right)\right) \times \\
& \left(\lambda_{j}-\frac{\left(\lambda_{j}\right)^{2}}{\lambda_{j}+\lambda_{\beta}}-\frac{\left(\lambda_{j}\right)^{2} \lambda_{\beta}}{\left(\lambda_{j}+\lambda_{\beta}\right)^{2}}\right) .
\end{aligned}
$$

There are two fuzzy parameters on the right side of each of the formulas (8) and (9); thus since the expressions are complex, uncertainty of ${ }^{h i} P_{(j+1) i}$ and ${ }^{s t} P_{\beta(i+1)}$ is not significantly bigger than the uncertainty of $\lambda_{\beta}, \lambda_{j}$ or $\lambda_{j+1}$.

The solution in the analytical form of the equation (5) $\left({ }^{o p t} t_{\beta(i+1)}^{u}\right)$ can't be found in this case: the task is solved by digital methods, applying fuzzy numbers comparison operations discussed in Chapter 3.

Expenditures (losses) through demurrage (waiting time):

$$
W_{W j(j+r) i}=W_{T} t_{j(j+r) i},
$$

when

$t_{j(j+r) i}$ - is the time from the corridor of the train $\beta$ after the train $\mathrm{j}$ up to the corridor after the train $(j+r)$;

$W_{T}$-are losses through demurrage of the train $\beta$ in a time unit.
Optimum traffic schedule of the additional train $(\beta)$ can be found applying the algorithms for the determination of the shortest chain.

\section{Fuzzy Algorithm for the Determination of the Shortest Chain}

A classical problem for the determination of the shortest chain is formulated in the following way: the known graph is:

$$
G=(V, A, L)
$$

$V=\{v\}=\{1,2, \ldots, n\}-$ a set of vertexes (their indices);

$A=\{a\}=\left\{\left.(x, y)\right|_{x \neq y}\right\}, x, y \in\{\mathrm{v}\}-$ a set of $\operatorname{arcs}(\mathrm{x}-$ index of the vertex from which the arc comes out, $y-$ index of the vertex to which the arc comes);

$L=\left\{\left.l(x, y)\right|_{x \neq y}>0\right\}-$ lengths (prices) of arcs.

To find a chain ${ }^{o} S_{b e} \in\left\{{ }^{k} S_{b e}\right\}$ in this graph between the initial vertex $b \in\{v\}$ and the end vertex $e \in\{v\}$, so that

$$
\sum_{u \in \in^{k} S_{b e}} l(x, y) \rightarrow \min _{k} .
$$

The analyzed problem differs from the classical one because a set of vertexes $V$ or (and) a set of $\operatorname{arcs} A$ are the fuzzy sets or (and) lengths (prices) of the $\operatorname{arcs} l(x, y)$ are expressed in fuzzy numbers. Thus the known graph is:

$$
\begin{aligned}
& \Gamma=(V, A, L) \\
& V=\left\{\mu_{v} / \nu\right\}=\left\{\mu_{1} / 1 . \mu_{2} / 2, \ldots, \mu_{n} / n\right\} \\
& \qquad 0 \leq \mu_{\nu} \leq 1 ; \\
& A=\{a\}=\left\{\mu_{(x, y)} /\left.(x, y)\right|_{x \neq y}\right\}, x, y \in\{\nu\} \\
& 0 \leq \mu_{(x, y)} \leq 1 ; \\
& L=\left\{\left.\mu_{1(x, y)}(t)\right|_{x \neq y}\right\}, x, y \in\{v\} \\
& 0 \leq \mu_{l(x, y)}(t) \leq 1,0 \leq t<\infty . \\
& \mu_{\nu}-\text { certainty factor of the graph } v \text { vertex; } \\
& \mu_{(x, y)} \text { - certainty (membership) factors of the graph }
\end{aligned}
$$
arcs; (price).

$\mu_{l(x, y)}(t)$ - membership functions of the arc length

In this case solution of the problem of the search of the shortest chain is uncertain:

$\left(\mu_{s} /{ }^{o} S_{b e}, \mu_{\mathrm{s}}(t)\right)$,

$\mu_{s}$ - factor of the existence (certainty) of the optimum chain ${ }^{o} S_{b e}$ between the vertexes $b$ and $e$;

$\mu_{s}(t)$ - length (price) membership function of the shortest chain ${ }^{o} S_{b e}$.

$\mu_{\mathrm{s}}$ depends on the chosen type of the fuzzy logic: min-max or the probability logic. 
In the first case

$$
\mu_{\mathrm{s}}=\min _{\nu \in^{o} S_{b e},(x, y) \in^{\circ} S_{b e}}\left\{\mu_{v}, \mu_{(x, y)}\right\},
$$

in the second case -

$$
\mu_{\mathrm{s}} \prod_{v \in \in^{0} S_{b e}} \mu_{v} \prod_{(x, y) \in^{o} S_{b e}} \mu_{(x, y)} .
$$

Thus the solution of the fuzzy problem of the search of the shortest chain is uncertain according to two parameters:

1) length (price) of the shortest chain;

2) factor of existence (reality) of the shortest chain.

Optimum solution according to both these parameters could be found only after formation of the complex factor that includes both mentioned parameters [24]. If there is no such (complex) factor, then the optimization is carried out only according to one of the said parameters: either looking for the chain with the biggest existence factor $\mu_{\mathrm{S}}$ (disregarding a length of the chain (price)); or the chain length (price) is minimized, disregarding the existence factor $\mu_{\mathrm{s}}$ of this chain. This case is closer (in the sense of its formulation) to the classical problem for the determination of the shortest chain (here it will be shown that the problem can be solved applying the same algorithm in both cases). Thus in this case it is enough to adapt the classical algorithm for the determination of the shortest chain for fuzzy initial data. It is reasonable to apply such classical algorithms in which the result is obtained with the least possible number of operations: uncertainty in fuzzy algorithms is increasing when the number of operations with the initial and intermediate data is increasing; multiplication and division operations are especially undesirable. Almost the most convenient algorithm for the determination of the shortest chain in this case could be the algorithm of FordFulkerson [5]. Its fuzzy version according to the succession of the procedures does not differ from the determinate one: only the form of initial data presentation and their processing procedures change.

\section{Beginning}

Double element index $\left(b, V_{b}=0\right)$ is given for the initial vertex $b$, and for all other vertexes $(i \neq b)$ - indices $\left(0, V_{i}=\infty\right)$.

1. It is searched for vertexes $j$, where

$$
V_{j}>V_{i}+\mu_{l(i, j)}(t)
$$

Index of every such vertex is changed with a new one

$$
\left(i, V_{j}=V_{i}+\mu_{l(i, j)}(t)\right)
$$

2. Procedure 1 is repeated until it is applicable.

3. The shortest chain is found according to the first elements of the double-element indices (because they specify the vertex from which the arc belonging to the shortest chain comes).

\section{End}

The main problem of the discussed algorithm is the comparison (ranking) of the fuzzy numbers (item 1 of the algorithm):

$$
V_{j .} \stackrel{\sum}{\equiv} V_{i}+\mu_{l(i, i)}(t)
$$

It should be noted that $V_{i}, V_{j}\left(\right.$ except $\left.V_{i=b}=0\right)$ also are fuzzy numbers.

There are several fuzzy numbers ranking criteria suggested in literature [6-14]. It is reasonable to apply the criteria that evaluate membership function form. E.g. $[8,12]$ :

$$
\begin{aligned}
& H_{2}(A, B)=\int_{0}^{1} M\left(A_{\alpha}\right) d \alpha-\int_{0}^{1} M\left(B_{\alpha}\right) d \alpha, \\
& M\left(A_{\alpha}\right)=\frac{1}{2}\left(\inf _{a \in A_{\alpha}} a-\sup _{a \in A_{\alpha}} a\right),
\end{aligned}
$$

$A_{\alpha}-$ a level set of the fuzzy number $A$.

$H_{2}(A, B) \geq 0 \Rightarrow A \geq B$.

$$
H_{3}(A, B)=\frac{\int_{a_{1}}^{a_{2}} \mu_{A}(a)\left(\int_{b_{1}}^{a} \mu_{B}(b) d b\right) d a}{\int_{a_{1}}^{a_{2}} \mu_{A}(a) d a \int_{b_{1}}^{b_{2}} \mu_{B}(b) d b},
$$

when $a_{1}=\inf _{a \in A_{0}} a ; a_{2}=\sup _{a \in A_{0}} a ; b_{1}$ and $b_{2}$ the sense is an analogous one.

$$
H_{3}(A, B) \geq 0,5 \Rightarrow A \geq B
$$

If a chain with the biggest existence factor $\mu_{S}$ is searched for (disregarding the chain length (price)), it is enough to exchange membership functions of the arc $\{(x$, $\left.y)_{x \neq y}\right\}, x, y \in\{v\}$ length (price) $\mu_{l\left(x_{1} y\right)}(t)$ with existence (reality) indices.

In case of min-max logic:

$$
\begin{aligned}
& \min \left\{\mu_{(x, y)}, \mu_{y}\right\}, \quad \text { when } x \neq b ; \\
& \mu_{a(p, y)}(t) \rightarrow \min \left\{\mu_{b}, \mu_{(b, y)}, \mu_{y}\right\} ;
\end{aligned}
$$

in case of probability logic:

$$
\begin{aligned}
& \mu_{(x, y)} \times \mu_{y}, \text { when } x \neq b, \\
& \mu_{l(p, y)}(t) \rightarrow \mu_{b} \times \mu_{(b, y)} \times \mu_{y} .
\end{aligned}
$$

Afterwards it is possible to apply the same algorithm.

Optimum traffic schedule of the additional train $(\beta)$ can be found applying the analyzed algorithm, only performing its item 1; indices of the vertical arcs $V_{M}$ are calculated according to the formula (4), and indices of the horizontal arcs - according to the formula 


$$
\begin{aligned}
& V_{W_{j}(j+1) i}={ }^{s t} P_{\beta i} W_{T} t_{j(j+1)}+\left(1-{ }^{s t} P_{\beta i}\right) \times \\
& \left.\left(W_{T}\right) t_{j(j+1)}+{ }^{s t} W_{i}\right),
\end{aligned}
$$

if the first step was made vertically, and according to the formula (10) - if the first step was made horizontally.

In this algorithm all data, except $\lambda_{\beta}, \lambda_{j}$ and $\lambda_{j+1}$ are considered to be determined (precise ones). Fuzzy parameters $\lambda_{\beta}, \lambda_{j}, \lambda_{j+1}$ may be presented in their membership functions $\left.\mu\left(\lambda_{u}\right)\right|_{\mathrm{u}}={ }_{\mathrm{j}, \beta_{\mathrm{B}}(\mathrm{j}+1) \text {. However such }}$ form for an expert (regulator) may be uncomfortable: it is easier for him to operate in linguistic variables. It is possible to use linguistic variables - "train's accuracy", instead of the fuzzy parameters $\lambda$ :

$$
\lambda_{u}^{2} \Delta \in[0,1], u=j, \beta,(j+1) \text {. }
$$

For this it is possible to use a fuzzifier of the train accuracy presented in Fig 3.

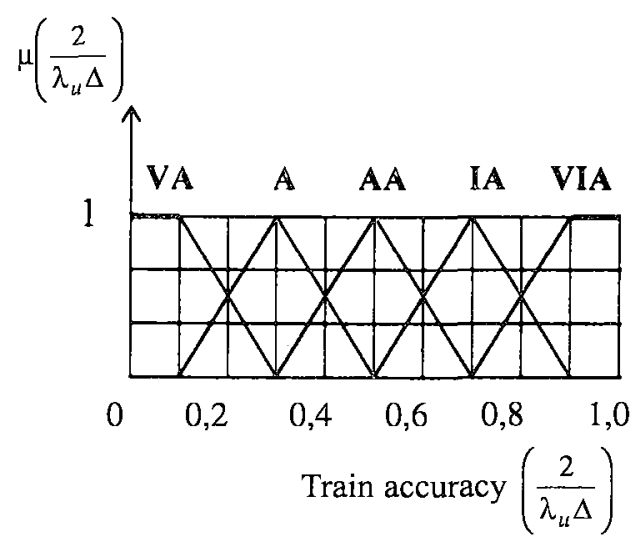

Fig 3. A fuzzifier of the train accuracy

VA - very accurate; A - accurate; AA - average accuracy; IA - inaccurate; VIA - very inaccurate

In this case the procedure of re-calculation of the linguistic variable terms should be provided (VA, A, AA, IA, VIA) into the fuzzy parameters $\lambda_{u}$ with the membership functions $\mu\left(\lambda_{u}\right)$ in the algorithm of the search of an optimum schedule of the additional train $\beta$.

\section{Conclusions}

1. A fuzzy algorithm for the determination of the shortest chain differs from the regular ones only in number comparison operators. The problem arises only because there is no uniform fuzzy number comparison criterion. It is purposeful to apply the criteria that evaluate a membership function form.

2. Problems of the traffic correction lead to the problems of the "shortest chain" and can be solved applying modified algorithms of the shortest chain search.

3. Traffic schedule correction problem is a mixed one: it is purposeful to make a part of calculations applying the regular methods, a part of them - by statistical ones, and another part - by expert (fuzzy) ones.

\section{References}

1. The Expenses for Freight Transportation per Railway Net Lines (Затраты на грузовые перевозки по участкам сети железньгх дорог). Edit. M. E. Mandrikov. Moscow: Transport, 1991. 223 p. (in Russian).

2. Ramsha Prabhu, T.; Vizayakumar, K. Technology choice using FDHM: A case of iron-making technology. IEEE Transactions of Engineering Management, Vol 48, 2001, p. 209-210.

3. Chan, F. T. S.; Chan, H. K.; Kazerooni, A. An intelligent fuzzy decision system for a flexible manufacturing system with multidecision points. In: Proceedings of the Mechanical Engineers, Vol 216 (7), 2002, p. 1021-1031.

4. Liao, T. Warren. A fuzzy multicriteria decision-making method for material selection. Journal of Manufacturing Systems, Vol 15 (1), 1966, p. 1-12.

5. Ford, L. R. Ir.; Fulkerson, D. R. Flows in Networks. Princeton University Press, 1962.

6. Baas, S. M.; Kwakernaak, H. Rating and Ranking of Multiple-Aspect Alternatives Using Fuzzy Sets. Automatica, Vol 13 (1), 1977, p. 47-58.

7. Baldwin, J. F.; Guild, N. C. F. Comparison of Fuzzy Sets on the Same Decision Space. Fuzzy Sets and Systems, Vol 2, 1979, p. 213-233.

8. Yager, R. A Procedure for Ordering Fuzzy Subsets of the Unit Interval. Information Sciences, Vol 24, 1981, p. 143 161.

9. Efstathiou, J.; Tong, R. M. Ranking Fuzzy Sets: A Decision Theoretic Approach. IEEE Transactions on Systems, Man and Cybernetics, Vol 12, 1982, p. 655-659.

10. Dubois, D.; Prade, H. Ranking of Fuzzy Numbers in the Setting of Possibility Theory. Information Sciences, Vol 30, 1983, p. 183-224.

11. Buckley, J. J. A Fuzzy Ranking of Fuzzy Numbers. Fuzzy Sets and Systems, Vol 33, 1989, p. 119-121.

12. Borisov, A. N. a.o. The Models of decision-making ground a linguistic variable (Модели принятия решгений на основе лингвистической переменной). Riga: Zinatne, 1982. 256 p. (in Russian).

13. Jason, Fisher. Make fuzzy comparisons using the Soundex algorithm in ASP. Active Server Developer's Journal, Feb. 2002, p. 7-11.

14. Prodanovic, P.; Simonovic, S. P. Comparison of fuzzy set ranking methods for implementation in water resources decision-making. Canadian Journal of Civil Engineering, Vol 29 (5), Ottawa, 2002, p. 692-701. 